\title{
Rapid Identification of Escherichia coli Colistin-Resistant Strains by MALDI-TOF Mass Spectrometry
}

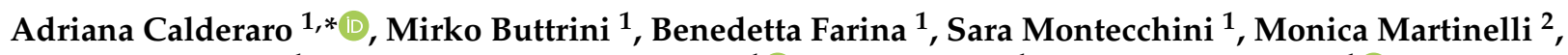 \\ Federica Crocamo ${ }^{1}$, Maria Cristina Arcangeletti ${ }^{1} \mathbb{C}$, Carlo Chezzi ${ }^{1}$ and Flora De Conto ${ }^{1} \mathbb{C}$ \\ 1 Department of Medicine and Surgery, University of Parma, Viale A. Gramsci 14, 43126 Parma, Italy; \\ mirko.buttrini@unipr.it (M.B.); benedetta.farina@studenti.unipr.it (B.F.); sara.montecchini@unipr.it (S.M.); \\ federica.crocamo@unipr.it (F.C.); mariacristina.arcangeletti@unipr.it (M.C.A.); carlo.chezzi@unipr.it (C.C.); \\ flora.deconto@unipr.it (F.D.C.) \\ 2 Unit of Clinical Microbiology, University Hospital of Parma, Viale A. Gramsci 14, 43126 Parma, Italy; \\ mmartinelli@ao.pr.it \\ * Correspondence: adriana.calderaro@unipr.it; Tel.: +39-0521-033499; Fax: +39-0521-993620
}

\section{check for} updates

Citation: Calderaro, A.; Buttrini, M.; Farina, B.; Montecchini, S.; Martinelli, M.; Crocamo, F.; Arcangeletti, M.C.; Chezzi, C.; De Conto, F. Rapid Identification of Escherichia coli Colistin-Resistant Strains by MALDI-TOF Mass Spectrometry. Microorganisms 2021, 9, 2210. https://doi.org/10.3390/ microorganisms 9112210

Academic Editor: Susann Teneberg

Received: 17 September 2021

Accepted: 21 October 2021

Published: 24 October 2021

Publisher's Note: MDPI stays neutral with regard to jurisdictional claims in published maps and institutional affiliations.

Copyright: (c) 2021 by the authors. Licensee MDPI, Basel, Switzerland. This article is an open access article distributed under the terms and conditions of the Creative Commons Attribution (CC BY) license (https:// creativecommons.org/licenses/by/ $4.0 /)$.

\begin{abstract}
Colistin resistance is one of the major threats for global public health, requiring reliable and rapid susceptibility testing methods. The aim of this study was the evaluation of a MALDI-TOF mass spectrometry (MS) peak-based assay to distinguish colistin resistant (colR) from susceptible (colS) Escherichia coli strains. To this end, a classifying algorithm model (CAM) was developed, testing three different algorithms: Genetic Algorithm (GA), Supervised Neural Network (SNN) and Quick Classifier (QC). Among them, the SNN- and GA-based CAMs showed the best performances: recognition capability (RC) of $100 \%$ each one, and cross validation (CV) of $97.62 \%$ and $100 \%$, respectively. Even if both algorithms shared similar RC and CV values, the SNN-based CAM was the best performing one, correctly identifying 67/71 (94.4\%) of the E. coli strains collected: in point of fact, it correctly identified the greatest number of colS strains $(42 / 43 ; 97.7 \%)$, despite its lower ability in identifying the colR strains $(15 / 18 ; 83.3 \%)$. In conclusion, although broth microdilution remains the gold standard method for testing colistin susceptibility, the CAM represents a useful tool to rapidly screen colR and colS strains in clinical practice.
\end{abstract}

Keywords: colistin resistance; MALDI-TOF MS; Gram-negative bacteria

\section{Introduction}

Antimicrobial resistance is one of the major threats for global public health, since many pathogens are developing resistance mechanisms to almost all currently available antimicrobial drugs [1-3]. This phenomenon is mostly related to the misuse and overuse of antimicrobials, which led to the emergence of multidrug-resistant (MDR), extensivelydrug-resistant (XDR) and pan-drug-resistant bacteria [4-7]. In particular, infections caused by resistant Gram-negative bacteria, such as Enterobacteriaceae, Pseudomonas aeruginosa and Acinetobacter baumannii, are a broad matter of concern, because of the ineffectiveness of conventional treatments and the lack of new antimicrobial agents against them $[1,3]$. Therefore, the occurrence and spread of resistant bacterial strains prompted the re-evaluation of polymixins (colistin and polymixin B), an old class of cationic, cyclic-polypeptide antibiotics $[6,8]$, whose clinical use was previously limited for their reported nephrotoxicity and neurotoxicity [9-11]. To date, colistin is considered a "last resort" antibiotic, namely a valid alternative to the classic antimicrobial agents ineffective against MDR Gram-negative pathogens $[1,3,6,8]$. Given its saving role against the life-threatening MDR and XDR bacterial infections, colistin was largely and recklessly employed both in human and veterinary medicine, resulting in the emergence of colistin-resistant pathogens, mainly Gram-negative bacteria $[1,3,6,12]$. Commonly, colistin-superbugs are the phenotypic expression of regulatory or mutational events in chromosomal genes [13-15]. However, resistance to this 
antibiotic can also be acquired by a plasmid-mediated strategy involving the mobile colistin resistance $(m c r)$ genes (mcr1-mcr10) [3,16-19]. The discovery of the $m c r$ genes on mobile genetic elements raised alarm given the possibility of their rapid dissemination by horizontal transfer $[19,20]$; therefore, reliable methods to detect colistin resistance are urgently needed [21-23]. Among the different laboratory techniques for testing the colistin susceptibility, the phenotypic ones, such as the disc diffusion and the gradient tests, are not very adequate because of the long turnaround time required, the low $\mathrm{mcr}$ sensitivity and specificity and the poor diffusion in agar of this drug [24,25]. Thus, the European Committee on Antimicrobial Susceptibility Testing (EUCAST) and the Clinical and Laboratory Standards Institute (CLSI) recommend the use of the broth microdilution (BMD), as the reference method to test colistin susceptibility among Gram-negative bacteria $[25,26]$. Even if BMD is considered the gold standard technique in determining the minimal inhibitory concentration (MIC) values of colistin in clinical microbiology laboratories, it is laborious and time consuming [24,25]. On the other hand, faster tools than BMD such as the automated MIC-determining systems (i.e., MicroScan, Sensititre, MICRONAUT-S, BD Phoenix, Vitek 2) do not meet the recommendations of EUCAST and CLSI, since they show low agreement with the reference test and, additionally, high rates of false susceptibility results $[22,24,27]$. Nevertheless, reliability and rapidity in detection of colistin resistance are crucial for antimicrobial stewardship and could be achieved by fast and reliable methods, such as MALDI-TOF MS [28]. This technique is already employed in clinical microbiology laboratories for the phenotypic identification of bacterial and fungal strains and its potentialities in predicting the antimicrobial resistance are being studied [26,29,30]. In particular, the MALDI-TOF MS approach for testing polymixins-resistance is based on the detection of biomarkers associated with the modified lipid A, which is the phenotypic result of both chromosomal and plasmid encoded resistance to colistin in Gram-negative bacteria. Therefore, given the inherent negative charge of the lipid A, several studies aimed to create MALDI-TOF MS tests to screen colistin resistance in Gram-negative bacteria by operating in a negative ion mode of the mass spectrometer [28,31-33]. However, to date, the negative ion mode is not currently and widely available on diagnostic routine mass spectrometers, since it works in a molecular mass range different from that used for the bacterial and fungal identification [32]. In this study, we describe an alternative approach for the identification of colistin resistance in Gram-negative bacteria by proposing a MALDI-TOF MS protein peak-based assay developed on the basis of spectra acquired in a positive linear mode embedded in the most widely used MALDI-TOF MS instrument available in clinical microbiology laboratories. The main aim of the study was to create a classifying algorithm model (CAM) able to rapidly detect and identify the colistin-resistant strains in clinical practice, in order to shorten the turnaround time by a simple and inexpensive tool.

\section{Materials and Methods}

\subsection{Bacterial Strains}

A total of 104 Gram-negative bacteria were included in this study: 71 Escherichia coli (Ec) strains (53 and 18 of human and veterinary origin, respectively) and 33 control other than E. coli strains, 15 Klebsiella pneumoniae (1 of animal origin), 9 Pseudomonas aeruginosa, 4 Acinetobacter baumanii, 5 Achromobacter xylosoxidans. The human strains were collected at the Unit of Clinical Microbiology of the University Hospital of Parma (81 strains) and at the Unit of Microbiology and Virology of the Hospital of Piacenza (4 strains) and the animal strains at the Department of Veterinary Science of Parma (Italy).

\subsection{Colistin Susceptibility Testing}

Colistin minimal inhibitory concentration (MIC) was determined by BMD (Liofilchem, Roseto degli Abruzzi, Teramo, Italy), following the manufacturer's instructions. Results were interpreted using Clinical and Laboratory Standards Institute (CLSI) breakpoints. 


\subsection{MALDI-TOF MS Protein Peak-Based Assay}

For testing colistin resistance by MALDI-TOF MS, a protein extraction protocol was performed. All the bacterial strains were cultured on horse blood agar (Kima, Italy) plates and incubated at $37^{\circ} \mathrm{C}$ for $24 \mathrm{~h}$; then, the isolated colonies were used to obtain a 3 McFarland bacterial suspension in sterile double-distilled water. An aliquot of $300 \mu \mathrm{L}$ of the bacterial suspension was added to $900 \mu \mathrm{L}$ of absolute ethanol, homogenized by vortex for $20 \mathrm{~s}$ and then centrifuged at $14.000 \times g$ for $2 \mathrm{~min}$. The supernatant was discharged, and the pellet was dried for $5 \mathrm{~min}$ under a laminar flow cabinet at room temperature, then suspended in $15 \mu \mathrm{L}$ of $70 \%$ formic acid and $15 \mu \mathrm{L}$ of acetonitrile, and finally vortexed (20 s) and centrifuged $(14.000 \times g$ for $2 \mathrm{~min})$. One $\mu \mathrm{L}$ of this supernatant obtained by protein extraction protocol was transferred on a MALDI-TOF target plate (10 replicates for each strain), dried under a stem of air and then overlaid with $1 \mu \mathrm{L}$ of $\alpha$-Cyano-4hydroxycinnamic acid Matrix (HCCA-Bruker Daltonics, Bremen, Germany), solubilized in 30:70 (v:v) acetonitrile/trifluoroacetic acid 0.01\% (TA30 Organic Solvent). The dried spots were analysed by the Autoflex Speed mass spectrometer (Bruker Daltonics, Germany), previously calibrated with "Bruker Bacterial Test Standard" according to manufacturer's instructions and set in MBT_Standard method (positive linear mode, with $60 \mathrm{~Hz}$ laser frequency, ion source voltage $20 \mathrm{kV}$ and mass molecular range 2-20 kDa). The spectra acquisition was performed in different independent experiments, by different operators in different days, in manual mode, in different points of the well with a laser intensity ranging from 30 to $40 \%$, and an overall 1400 laser-shot, by 200 shot steps. The acquired spectra were baselined and smoothed by FlexAnalysis software (version 3.1, Bruker Daltonics), and only those with $>104$ intensity arbitrary units were used for further analysis. The normalized spectra were then loaded into the MALDI Biotyper software (version 3.1.66, Bruker Daltonics) to verify their validity, and to identify the bacterial species; the replicates identified with a $<2$-score value were discarded.

\subsection{Classifying Algorithm Model}

Based on BMD susceptibility results, the 71 E. coli strains were divided in two groups: "training set" and "test set". The "training set" included 10 E. coli strains, 5 colistin-resistant (colR-Ec) and 5 colistin-susceptible (colS-Ec), randomly selected; the "test set" included the remaining 61 E. coli strains.

To rapidly and correctly identify both the colistin-resistant (colR) strains and the susceptible (colS) ones, a classifying algorithm model (CAM) was developed by using the "training set". A total of 80 "training set" spectra (8 replicates/strain) were imported in ClinProTools software (version 3.0, Bruker Daltonics), in order to develop a CAM able to discriminate between the colR-Ec and the colS-Ec on the basis of their different protein profiles. The analysis was performed focusing on the molecular mass range $2-20 \mathrm{kDa}$ with a 4 signal-to-noise threshold, an 0.08 relative threshold base peak, and the "Shift Maximum Peak" parameter set up at 1000 parts per million (ppm). The software calculated an average spectrum of each isolate (single average spectrum), an average spectrum of both the classes considered (colR-Ec average spectrum and colS-Ec average spectrum) and an average spectrum based on all replicates of all analyzed isolates (cumulative average spectrum), and then provided a list of peaks potentially differentiating the colR-Ec strains and the colS-Ec ones. In addition, this spectra dataset ("training set") was analysed by Principal Component Analysis (PCA), an unsupervised hierarchical type of clustering, in order to visualize the homogeneity and heterogeneity of the protein spectra. The PCA results are called scores and are derived and displayed in various plots. The score output represents the original data mapped into the new coordinate system, which is defined by the Principal Components (PCs). Within the score plot, outlier spectra from a group or from several groups can be discovered and visualized. The outliers are spectra that are extreme or do not fit the PCA model. Independently from the PC coordinates, the score plots contain the same spectra number as the original data set. Moreover, the percentage of the "explained variance" of the single given PC was also reported. 
For the development of the CAM, the Genetic Algorithm (GA), the Quick Classifier (QC), and the Supervised Neural Network (SNN) algorithm-based models were applied to the preliminary pattern of protein peaks found by the software. Each algorithm performed a selection of different discriminating peaks in order to improve its classification performance. Each CAM was characterized by recognition capability (RC) and cross-validation (CV) values, parameters of the accuracy of the model. For the development of each algorithm, different combinations of parameters were evaluated and, for each one, the most performing in terms of RC and CV was reported. The reliability and the accuracy of each algorithm-based model were verified by performing an internal and an external validation. In particular, the "training set" were used for the internal validation, while the external validation was achieved with the "test set".

\subsubsection{Genetic Algorithm}

The Genetic Algorithm (GA) works on a population of peaks and selects the fittest peaks combinations that are the most relevant for the classification. At each step, the GA randomly selects peaks from the preliminary pattern of protein peaks found by the Software and uses them as "parents" to produce the "children" for the next generation. In this study, the parameters of this algorithm were set as follows. A maximum number of 15 peaks was chosen to be included in the model, with an automatic detection mode to determine the best number of peak combinations to integrate (npc) by applying the heuristic formula NPC $=100+($ Number of picked peaks $\times 20) /($ Maximal number of peaks in model +1$)$. Within the GA algorithm, the k-nearest neighbor $(\mathrm{k}-\mathrm{NN})$ classifier algorithm allowed us to obtain the final classification. The k-NN algorithm calculated the distances between the points in the n-dimensional space; each point corresponded to a spectrum whose area defined its coordinates. The number of neighbors, the mutation rate and the crossover rate were set at 7 (values among 3, 5, and 7), 0.2 (range values 0.0-1.0) and 0.5 , respectively. In ClinProTools, both the mutation rate and the crossover rate are advanced parameters. In particular, the mutation rate is the probability of a mutation, namely the random exchange of a peak within peak combinations by a randomly selected new one; on the other hand, the crossover rate is the likelihood of a crossover between peaks combinations.

\subsubsection{Quick Classifier}

The QuickClassifier (QC) is a univariate sorting algorithm. The class averages of the peak areas are stored in the model, together with statistical data such as the $p$-values at certain peak positions. For classification, the peak areas/intensities are sorted per peak and a weighted average over all peaks is calculated.

The QC automatically uses automatic peak detection to determine the best number of peaks to be integrated in the model (maximum is 25 peaks). The sort mode used for peak ranking and as weight was $p$-value obtained by Student's $t$-test/ANOVA.

\subsubsection{Supervised Neural Network}

The Supervised Neural Network (SNN) is a prototype-based classification algorithm that identifies some characteristic spectra for each class, which could be consider as prototypes of that class. In an initial step, a predefined number of prototypes are spread over the data space by using the Batch-Neural-Gas algorithm, which gives an optimal distribution of the prototypes over the data space in accordance with the data density properties. In a second step, the SNN optimizes the positions of the prototypes with respect to the class information (supervised) minimizing the empirical risk. Thereby, the used metric of the data space is adapted such that dimensions, which are relevant for the class separation, are higher weighted than dimensions that do not contribute to class separation. This procedure of optimizing prototype positions with a combined feature selection is applied iteratively for a predefined upper limit of steps. 
The SNN uses an automatic peak detection mode to determine the best number of peaks (maximum 25 peaks) to be integrated in the model and the automatic detection of prototype number was applied. The upper limit of cycles to run for optimizing the prototype positions was set at 2000 .

\subsection{Statistical Analysis}

Statistical Analysis was automatically performed by ClinProTools software. In particular, the $p$-value is obtained by both parametric and non-parametric statistical tests. With regards to parametric tests, the software automatically selected between Student's $t$-test (two classes involved) or ANOVA (more than two classes involved). Similarly, for non-parametric tests, the software automatically selected between Wilcoxon test (2 classes involved) or Kruskal-Wallis test (more than two classes involved).

The $p$-value was calculated by comparing each single average spectrum with the cumulative average spectrum, based on both parametric (Student's $t$-test) and non-parametric (Wilcoxon-W) statistical tests. The software considered the peaks associated to a $p$-value $\leq 0.05$ as potentially able to discriminate between the considered classes. However, since the best discriminating peaks are associated to low $p$-values, the significance threshold was set to 0.01 , in order to improve the performance.

\section{Results}

According to the colistin susceptibility testing results, among all E. coli isolates, 48 (33 human and 15 animal strains) were colistin-susceptible and 23 (20 human and three animal strains) were colistin-resistant (MIC $\geq 4 \mathrm{mg} / \mathrm{L}$ ). Both the colR-Ec strains and the colS-Ec strains were correctly identified at the species level by MALDI-TOF MS, with score values above 2.0. When the "training set" was used to develop the CAM, a list of 47 potential discriminating peaks (Supplementary Table S1) was obtained by the ClinProTools software. The additional PCA analysis of the spectra showed two different clusters referred to colR-Ec and colS-Ec strains (Figure 1).

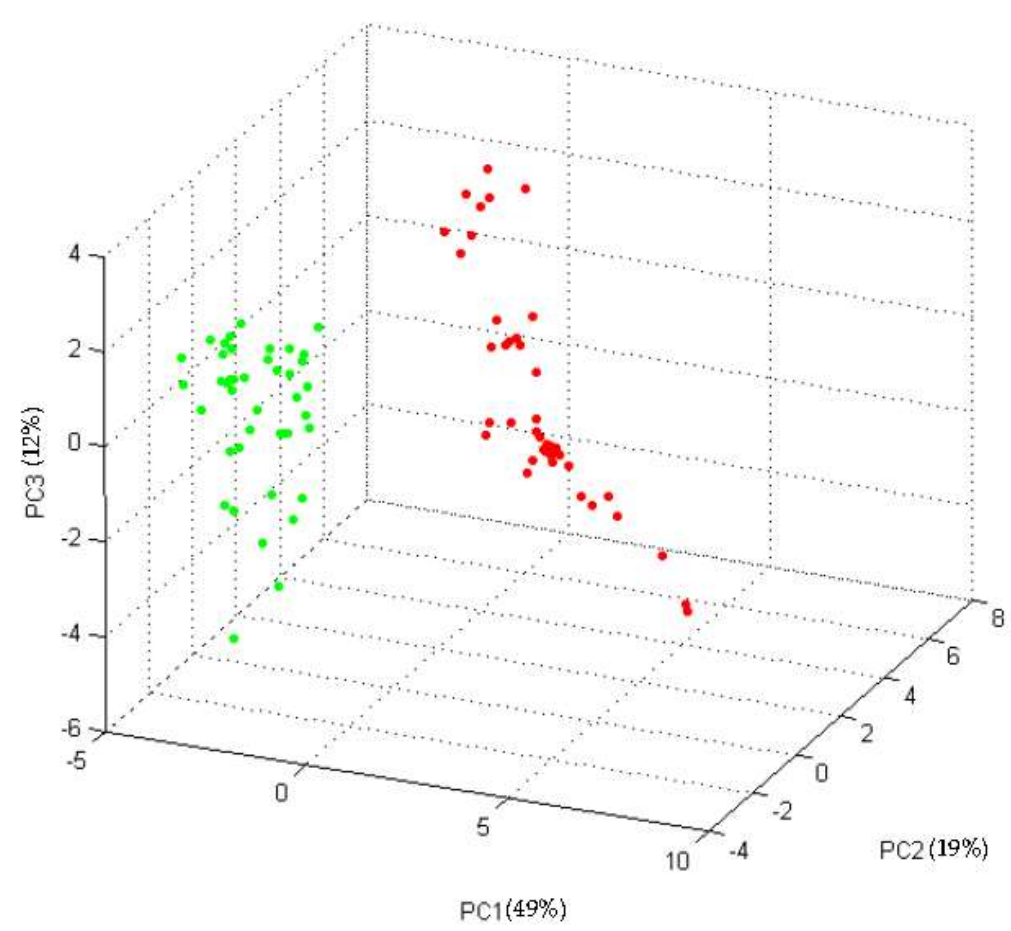

Figure 1. Three-dimensional plot of the spectra of the "training set" strains obtained by Principal Component Analysis (PCA) (dots of the same colour represent replicates of strains of the same class: colR-Ec strains in red and colS-Ec strains in green). For each principal component (PC), the percentage of the "explained variance" is reported in round bracket. 
In order to verify the reliability of these discriminating peaks, 61 different strains ("test set"), 43 colS-Ec and 18 colR-Ec were loaded into ClinProTools software and the PCA was created (Figure 2A,B, respectively).

A

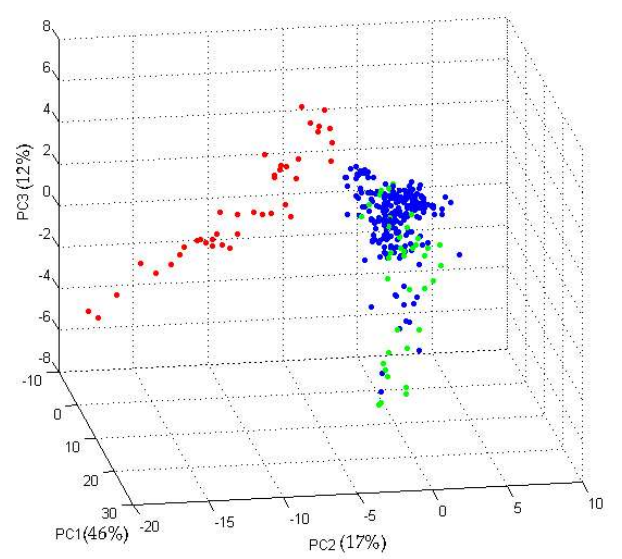

B

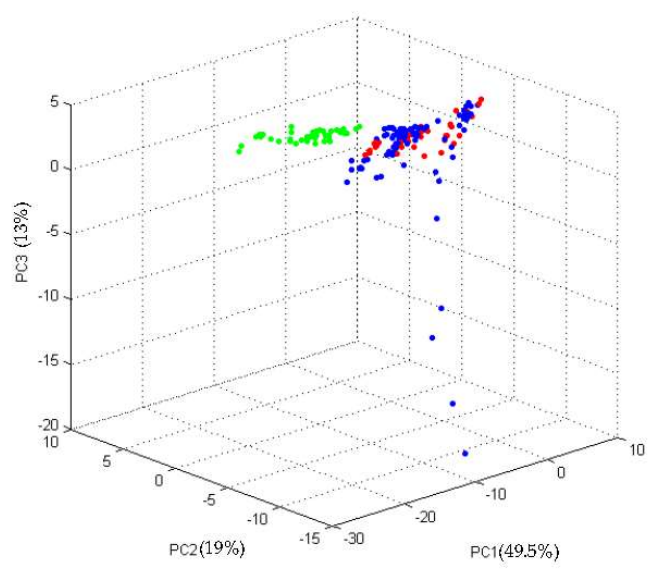

Figure 2. Three-dimensional plot of the spectra of the "training set" strains obtained by Principal Component Analysis (PCA) (colR-Ec strains in red and colS-Ec strains in green) in comparison to: (A) colS-Ec "test set" strains (blue) and (B) colR-Ec "test set" strains (blue) (dots of the same colour represent replicates of strains of the same class). For each principal component (PC), the percentage of the "explained variance" is reported in round bracket.

The "PCA-3D plot" obtained for colR-Ec showed that these strains clustered close to colR-Ec strains used as "training set" and were totally separated from colS-Ec strains. Similarly, the PCA obtained for colS-Ec strains showed that these strains clustered close to colS-Ec strains used as "training set" and were totally separated from colR-Ec strains.

The three algorithms applied to the pattern of 47 peaks showed different performances in terms of RC, CV and list of peaks involved, as reported in Table 1.

Table 1. Performances and list of peaks of the different algorithms tested. GA Genetic Algorithm; SNN Supervised Neural Network; QC Quick Classifier; RC Recognition Capability; CV Cross Validation; Da Dalton; + peak involved in classifying algorithm model (CAM).

\begin{tabular}{|c|c|c|c|c|c|c|c|c|c|c|c|c|c|c|}
\hline \multirow{2}{*}{$\begin{array}{l}\text { Algorithms } \\
\text { Model }\end{array}$} & \multirow{2}{*}{$\mathrm{RC}(\%)$} & \multirow{2}{*}{ CV $(\%)$} & \multicolumn{12}{|c|}{ Peaks Used for Classification (Da) } \\
\hline & & & 4177 & 4365 & 4440 & 4449 & 4498 & 5612 & 6257 & 6283 & 8330 & 8878 & 9066 & 9715 \\
\hline GA & 100 & 100 & + & + & + & & + & & + & & + & + & & \\
\hline SNN & 100 & 97.62 & + & & + & & & + & + & + & & & + & + \\
\hline $\mathrm{QC}$ & 98.81 & 94.21 & & & & + & & & & + & & + & & \\
\hline
\end{tabular}

The GA-based model automatically selected seven peaks with a $p$-value $<0.008$ for both statistical tests. Both the RC and CV values were $100 \%$. As concern the internal and the external validations, this CAM correctly classified $100 \%$ and $82 \%(50 / 61)$ of the E. coli strains included in the "training set" and in the "test set", respectively. In particular, $88.9 \%$ $(16 / 18)$ of colR-Ec and 79\% (34/43) of the colS-Ec strains of the "test set" were correctly classified (Table 2).

The SNN-based model automatically selected seven peaks associated to a $p$-value $<0.000001$ for both statistical tests, with $100 \%$ and $97.62 \%$ of RC and CV, respectively. Concerning the internal validation, $100 \%$ of the colR-Ec and colS-Ec "training set" strains were correctly classified (Table 2); conversely, this CAM correctly classified $93.4 \%$ of the strains included in the "test set" (57/61): 83.3\% (15/18) and 97.7\% (42/43) of the colR-Ec and colS-Ec external control strains, respectively, were correctly classified (Table 2). 


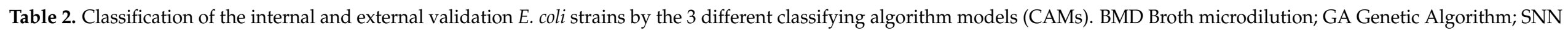
Supervised Neural Network; QC Quick Classifier; colR colistin-resistant; colS colistin-susceptible.

\begin{tabular}{|c|c|c|c|c|c|c|c|c|c|c|c|}
\hline \multirow{3}{*}{ Set of Strains } & \multirow{3}{*}{ BMD } & \multirow{3}{*}{ No. } & \multicolumn{9}{|c|}{ CAM Classification } \\
\hline & & & \multicolumn{3}{|r|}{ GA } & \multicolumn{3}{|c|}{ SNN } & \multicolumn{3}{|r|}{ QC } \\
\hline & & & colR & cols & Correctly Classified (\%) & colR & cols & Correctly Classified (\%) & colR & cols & Correctly Classified (\%) \\
\hline \multirow{3}{*}{$\begin{array}{c}\text { Internal } \\
\text { validation }\end{array}$} & colR & 5 & 5 & & $5 / 5(100)$ & 5 & & $5 / 5(100)$ & 5 & & $5 / 5(100)$ \\
\hline & cols & 5 & & 5 & $5 / 5(100)$ & & 5 & $5 / 5(100)$ & & 5 & $5 / 5(100)$ \\
\hline & & 10 & & & $10 / 10(100)$ & & & $10 / 10(100)$ & & & $10 / 10(100)$ \\
\hline \multirow{2}{*}{$\begin{array}{c}\text { External } \\
\text { validation }\end{array}$} & colR & 18 & 16 & 2 & $16 / 18(88.9)$ & 15 & 3 & $15 / 18(83.3)$ & 17 & 1 & $17 / 18(94.4)$ \\
\hline & & 61 & & & $50 / 61(82)$ & & & $57 / 61(93.4)$ & & & $45 / 61(73.8)$ \\
\hline Overall & & 71 & & & $60 / 71(84.5)$ & & & $67 / 71(94.4)$ & & & $55 / 71(77.5)$ \\
\hline
\end{tabular}


Finally, the QC-based model automatically selected three peaks with a $p$-value $<0.000001$ for both statistical tests, classifying strains with overall RC (98.81\%) and CV (94.21\%) values lower than the other two algorithm-based models tested. The QC-based CAM correctly classified $100 \%$ of the internal control strains and $73.8 \%$ of the strains included in the "test

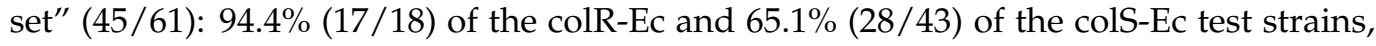
respectively, were correctly classified (Table 2 ).

With regard to the absolute number of errors, GA-based CAM failed to classify 11 strains out of the 61 included in the "test set", while SNN-based CAM failed to classify four strains, and QC-based CAM 16.

Among the 18 colR-Ec strains, one human strain was wrongly classified as colS-Ec by each CAM tested; similarly, among the 43 colS-Ec strains, one animal strain was wrongly classified as colR-Ec by the three CAMs.

The pattern of peaks used for the classification by the SNN-based CAM included two peaks (9066 Da and 9715 Da) (Figure 3), not included in the GA and QC algorithms' list of peaks.

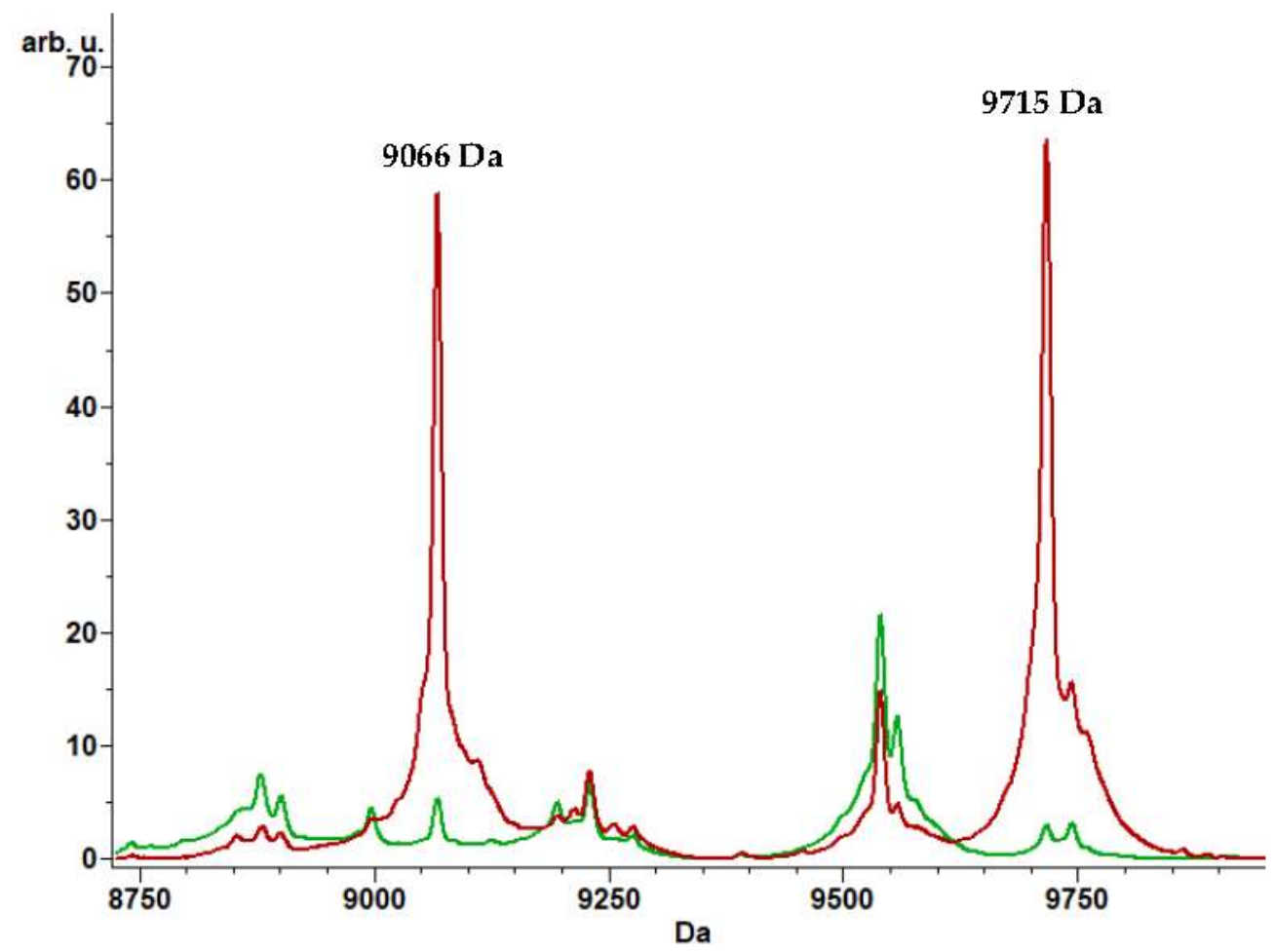

Figure 3. Peaks used for the classification by the SNN-based CAM (9066 Da and $9715 \mathrm{Da}$ ) not involved by the GA and QC algorithms (colR-Ec "training set" Average spectrum in red and colS-Ec "training set" Average spectrum in green). arb. u. arbitrary units; Da dalton.

The molecular weights of these two peaks were found to correspond to those of a hypothetical protein related to the IncK2 carrying mcr-1 gene plasmid (Accession number ASO65104.1; 86 amino acids; molecular weight $9090 \mathrm{Da}$ ) and of a transcriptional regulator linked to a Plasmid-mediated mcr-1 (pICBEC7Pmcr) in a carbapenem-susceptible E. coli strain (Accession number OKO56538.1; 85 amino acids; molecular weight $9700 \mathrm{Da}$ ), respectively.

These three different CAMs patterns of peaks were evaluated also with colR and colS strains (16 and 17 strains, respectively) other than E. coli (Table 3). GA-based CAMs correctly classified $45.5 \%$ (15/33) of strains: $50 \%(8 / 16)$ of colR and $41.2 \%(7 / 17)$ of colS strains. SNN-based CAM correctly classified $48.5 \%(16 / 33)$ of strains: $50 \%(8 / 16)$ of colR and $47 \%(8 / 17)$ of colS strains. QC-based CAM classified all strains as colR. 


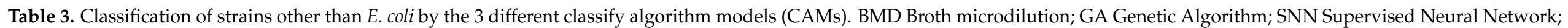
QC Quick Classifier; colR colistin-resistant; colS colistin-susceptible.

\begin{tabular}{|c|c|c|c|c|c|c|c|c|c|c|c|}
\hline \multirow{3}{*}{ Strains } & \multirow{3}{*}{ BMD } & \multirow{3}{*}{ No. } & \multicolumn{9}{|c|}{ CAM Classification } \\
\hline & & & \multicolumn{3}{|r|}{ GA } & \multicolumn{3}{|r|}{ SNN } & \multicolumn{3}{|r|}{$\mathrm{QC}$} \\
\hline & & & colR & cols & Correctly Classified (\%) & colR & cols & Correctly Classified (\%) & colR & cols & Correctly Classified (\%) \\
\hline K. pneumoniae & colR & 7 & 3 & 4 & $3 / 7(42.9)$ & & 7 & $0 / 7(0)$ & 7 & & $7 / 7(100)$ \\
\hline P. aeruginosa & colR & 2 & 2 & & $2 / 2(100)$ & 2 & & $2 / 2(100)$ & 2 & & $2 / 2(100)$ \\
\hline A. baumannii & colR & 2 & 2 & & $2 / 2(100)$ & 2 & & $2 / 2(100)$ & 2 & & $2 / 2(100)$ \\
\hline \multirow[t]{2}{*}{ A. xylosoxydans } & colR & 5 & 1 & 4 & $1 / 5(20)$ & 4 & 1 & $4 / 5(80)$ & 5 & & $5 / 5(100)$ \\
\hline & & 16 & & & $8 / 16(50)$ & & & $8 / 16(50)$ & & & $16 / 16(100)$ \\
\hline K. pneumoniae & cols & 8 & 1 & 7 & $7 / 8(87.5)$ & & 8 & $8 / 8(100)$ & 8 & & $0 / 8(0)$ \\
\hline P. aeruginosa & cols & 7 & 7 & & $0 / 7(0)$ & 7 & & $0 / 7(0)$ & 7 & & $0 / 7(0)$ \\
\hline \multirow[t]{2}{*}{ A. baumannii } & cols & 2 & 2 & & $0 / 2(0)$ & 2 & & $0 / 2(0)$ & 2 & & $0 / 2(0)$ \\
\hline & & 17 & & & $7 / 17$ (41.2) & & & $8 / 17$ (47) & & & $0 / 17(0)$ \\
\hline Overall & & 33 & & & $15 / 33(45.5)$ & & & $16 / 33(48.5)$ & & & $16 / 33(48.5)$ \\
\hline
\end{tabular}




\section{Discussion}

In the last decade, colistin has been proposed as a last-line antibiotic for the treatment of MDR Gram-negative infections.

However, despite its high capacity in bacterial killing, colistin resistance phenomenon is emerging as a result of mutations in the efflux pump operon, and in genes encoding lipid A. These resistance mechanisms to colistin have gained global attention and pose a new threat to public health $[26,33]$.

Furthermore, as widely reported by EUCAST and CLSI, the methods most frequently used in clinical microbiology laboratories for performing antimicrobial susceptibility testing (automated systems, E-test) are not reliable for detecting colistin resistance, concluding that BMD represents the reference method for colistin susceptibility testing.

More rapid, and cost-effective clinical microbiology technologies, such as MALDITOF MS, are currently being evaluated in order to overcome the use of BMD method and to obtain a reliable detection of colistin-resistant strains, by directly assessing the biochemical cause of resistance, the modification of lipid A [31]. However, the MALDI-TOF MS method for colistin resistance detection is usually performed in a linear negative-ion mode [31], not embedded in all instruments used in diagnostic laboratories, in a mass range $<2 \mathrm{kDa}$. This study aimed to detect colistin resistance in E. coli strains by MALDI-TOF MS in the linear positive-ion mode, within the mass range 2-20 kDa commonly used for the routine identification of bacteria and fungi [34-37]. Firstly, we created a CAM to automatically distinguish $E$. coli strains as colR or colS using $10 \mathrm{E}$. coli strains (5 colR and $5 \mathrm{colS}$ ), arbitrarily selected. The multivariate unsupervised statistical analysis, PCA, applied to this data set, showed the spectra replicates of the two different analyzed classes (colistin-resistant and colistin-susceptible) placed in two well separated clusters, suggesting the possible differentiation of these two classes based on the presence of specific peaks in the mass range $2-20 \mathrm{kDa}$. In particular, a pattern of 47 potential discriminating peaks was evaluated using three different algorithms-based CAMs (GA, SNN and QC). The three CAMs identified different patterns of discriminating peaks, which, however, showed similar RC $(100 \%, 100 \%$ and $98.81 \%$, respectively), and CV $(100 \%, 97.62 \%$ and $94.21 \%$, respectively) values. In addition, in all three cases a correct identification of the $100 \%$ of the internal control strains was obtained. Despite that GA-based CAM showed the highest values of RC and CV, it demonstrated a moderate ability to detect colS-Ec strains (34/43; $79 \%)$. On the contrary, the external validation of the QC-based CAM was not satisfactory, since only $73.8 \%(45 / 61)$ of the strains was correctly identified, and in particular only $65.1 \%$ $(28 / 43)$ of colS-Ec strains.

Among the three CAMs tested, even if it showed the lowest ability in identifying colREc strains $(15 / 18 ; 83.3 \%)$, the SNN-based model correctly identified the greatest number of colS-Ec strains $(42 / 43 ; 97.7 \%)$. The only colS-Ec strain wrongly classified by this CAM was also mis-identified by the other two.

However, if considering the absolute number of errors, GA-based CAM failed to classify 11 strains of the validation set, while SNN-based CAM failed to identify only four strains. The correct identification of the majority of the colS-Ec strains by SNN-based CAM could be due to the inclusion in the classification peak list of two peaks (9066 Da and $9715 \mathrm{Da}$ ) having a molecular weight similar to the two proteins related to a plasmid, which carries mcr-1 gene found in E. coli. The same reason could explain the failure of the same CAM in the classification of three colR strains, which may show a colistin resistance mechanism different from mcr-1.

Each CAM showed a limited capability in classifying Gram-negative colR and colS strains other than E. coli, likely due to the diversity between their species-specific protein profiles and those of $E$. coli, on which the development of the three CAMs was based. Therefore, a classification model is able to perform an intraspecific discrimination between colR and colS strains, which are probably classified on the basis of species-specific colistin resistance mechanisms, whose molecular effectors could have a molecular mass within the 2-20 kDa range. Although this approach is not based on the traditional colistin resistance 
biomarkers (i.e., modifications in lipid A, involving phosphoethanolamine), the development of a species-specific CAM allows a rapid screening of colR and colS strains on the basis of species-related discriminating peaks, and without resorting to the more time-consuming BMD method. As a matter of fact, although the conventional antibiotic susceptibility tests, such as BMD, allow the phenotypic characterization of resistant strains, they suffer from a higher time-to-result because of the long time required for the bacterial growth and the phenotypic expression of the resistance mechanism, consequently preventing a prompt and appropriate treatment.

\section{Conclusions}

In conclusion, the present study describes an alternative, rapid, simple to perform, inexpensive and reliable MALDI-TOF MS application for the identification of colR E. coli strains in routine diagnostic. Although no one of the CAM created in this study is $100 \%$ reliable for both the categories tested, this approach could represent an alert, at the same time as the bacterial identification, for the presence of colR strains, which, as suggested by CLSI and EUCAST, should be investigated using the gold standard method (BMD assay) for MIC determination or molecular approach to determine genetic resistance mechanisms.

Supplementary Materials: The following is available online at https:/ / www.mdpi.com/article/10 .3390/microorganisms9112210/s1 Table S1: list of potential discriminating peaks.

Author Contributions: Conceptualization, A.C. and C.C.; methodology, A.C., M.B. and M.M.; validation, A.C., M.B., B.F., S.M. and M.M.; formal analysis, M.B., B.F. and S.M.; investigation, M.B., B.F. and F.C.; data curation, A.C., M.B., B.F., S.M., M.M., F.C., M.C.A. and F.D.C.; writing-original draft preparation, A.C., M.B., B.F., S.M., M.M. and C.C.; visualization, A.C., M.B., M.M., M.C.A., C.C. and F.D.C.; supervision, A.C., M.C.A., C.C. and. F.D.C.; project administration, A.C., M.B., S.M., M.M., C.C.; funding acquisition, A.C. All authors have read and agreed to the published version of the manuscript.

Funding: This study was supported by the Ministry of University and Scientific Research Grant FIL, University of Parma, Parma, Italy and the grant "Fondo di finanziamento per le attività base di ricerca (FFABR)" from the Italian Ministry for the University and Research (Ministero dell'Università e della Ricerca, MUR).

Institutional Review Board: Not applicable. Only bacterial strains from laboratory collections were used in this study.

Informed Consent Statement: Not applicable. Only collected strains were used in this study.

Data Availability Statement: The data presented in this study are available in the manuscript and in the supplementary materials (Supplementary Table S1).

Acknowledgments: The Authors thank Maria Cristina Ossiprandi of the Department of MedicalVeterinary Sciences of the University of Parma and Giuliana Lo Cascio of the Unit of Microbiology and Virology of the Hospital of Piacenza for supplying some of the strains.

Conflicts of Interest: The authors declare no conflict of interest. A.C. is a Microorganisms's Editorial Board member.

\section{References}

1. Lim, L.M.; Ly, N.; Anderson, D.; Yang, J.C.; Macander, L.; Jarkowski, A., 3rd; Forrest, A.; Bulitta, J.B.; Tsuji, B.T. Resurgence of colistin: A review of resistance, toxicity, pharmacodynamics, and dosing. Pharmacotherapy 2010, 30, 1279-1291. [CrossRef] [PubMed]

2. WHO. Global Report on Surveillance 2014; 2014 AMR Rep; WHO: Geneva, Switzerland, 2014; pp. 1-8.

3. El-Sayed Ahmed, M.A.E.-G.; Zhong, L.-L.; Shen, C.; Yang, Y.; Doi, Y.; Tian, G.-B. Colistin and its role in the Era of antibiotic resistance: An extended review (2000-2019). Emerg. Microbes Infect. 2020, 9, 868-885. [CrossRef] [PubMed]

4. Levy, S.B.; Marshall, B. Antibacterial resistance worldwide: Causes, challenges and responses. Nat. Med. 2004, 10, S122-S129. [CrossRef] [PubMed]

5. Christaki, E.; Marcou, M.; Tofarides, A. Antimicrobial Resistance in Bacteria: Mechanisms, Evolution, and Persistence. J. Mol. Evol. 2020, 88, 26-40. [CrossRef] 
6. Sherry, N.; Howden, B. Emerging Gram negative resistance to last-line antimicrobial agents fosfomycin, colistin and ceftazidimeavibactam-Epidemiology, laboratory detection and treatment implications. Expert Rev. Anti. Infect. Ther. 2018, 16, 289-306. [CrossRef] [PubMed]

7. Prestinaci, F.; Pezzotti, P.; Pantosti, A. Antimicrobial resistance: A global multifaceted phenomenon. Pathog. Glob. Health 2015, 109, 309-318. [CrossRef] [PubMed]

8. Falagas, M.E.; Kasiakou, S.K. Colistin: The revival of polymyxins for the management of multidrug-resistant gram-negative bacterial infections. Clin. Infect. Dis. Off. Publ. Infect. Dis. Soc. Am. 2005, 40, 1333-1341. [CrossRef] [PubMed]

9. Brown, J.M.; Dorman, D.C.; Roy, L.P. Acute renal failure due to overdosage of colistin. Med. J. Aust. 1970, 2, 923-924. [CrossRef] [PubMed]

10. Koch-Weser, J.; Sidel, V.W.; Federman, E.B.; Kanarek, P.; Finer, D.C.; Eaton, A.E. Adverse effects of sodium colistimethate. Manifestations and specific reaction rates during 317 courses of therapy. Ann. Intern. Med. 1970, 72, 857-868. [CrossRef]

11. Ryan, K.J.; Schainuck, L.I.; Hickman, R.O.; Striker, G.E. Colistimethate toxicity. Report of a fatal case in a previously healthy child. JAMA 1969, 207, 2099-2101. [CrossRef] [PubMed]

12. Bialvaei, A.Z.; Samadi Kafil, H. Colistin, mechanisms and prevalence of resistance. Curr. Med. Res. Opin. 2015, 31, 707-721. [CrossRef]

13. Hamel, M.; Rolain, J.-M.; Baron, S.A. The History of Colistin Resistance Mechanisms in Bacteria: Progress and Challenges. Microorganisms 2021, 9, 442. [CrossRef] [PubMed]

14. Olaitan, A.O.; Morand, S.; Rolain, J.-M. Mechanisms of polymyxin resistance: Acquired and intrinsic resistance in bacteria. Front. Microbiol. 2014, 5, 643. [CrossRef] [PubMed]

15. Lee, H.; Roh, K.H.; Hong, S.G.; Shin, H.B.; Jeong, S.H.; Song, W.; Uh, Y.; Yong, D.; Lee, K. In Vitro Synergistic Effects of Antimicrobial Combinations on Extensively Drug-Resistant Pseudomonas aeruginosa and Acinetobacter baumannii Isolates. Ann. Lab. Med. 2016, 36, 138-144. [CrossRef] [PubMed]

16. Ling, Z.; Yin, W.; Shen, Z.; Wang, Y.; Shen, J.; Walsh, T.R. Epidemiology of mobile colistin resistance genes mcr-1 to mcr-9. J. Antimicrob. Chemother. 2020, 75, 3087-3095. [CrossRef]

17. Liu, Y.-Y.; Wang, Y.; Walsh, T.R.; Yi, L.-X.; Zhang, R.; Spencer, J.; Doi, Y.; Tian, G.; Dong, B.; Huang, X.; et al. Emergence of plasmid-mediated colistin resistance mechanism MCR-1 in animals and human beings in China: A microbiological and molecular biological study. Lancet Infect. Dis. 2016, 16, 161-168. [CrossRef]

18. Wang, C.; Feng, Y.; Liu, L.; Wei, L.; Kang, M.; Zong, Z. Identification of novel mobile colistin resistance gene mcr-10. Emerg. Microbes Infect. 2020, 9, 508-516. [CrossRef] [PubMed]

19. Wang, R.; van Dorp, L.; Shaw, L.P.; Bradley, P.; Wang, Q.; Wang, X.; Jin, L.; Zhang, Q.; Liu, Y.; Rieux, A.; et al. The global distribution and spread of the mobilized colistin resistance gene mcr-1. Nat. Commun. 2018, 9, 1179. [CrossRef]

20. Dolejska, M.; Papagiannitsis, C.C. Plasmid-mediated resistance is going wild. Plasmid 2018, 99, 99-111. [CrossRef]

21. Jayol, A.; Nordmann, P.; Lehours, P.; Poirel, L.; Dubois, V. Comparison of methods for detection of plasmid-mediated and chromosomally encoded colistin resistance in Enterobacteriaceae. Clin. Microbiol. Infect. Off. Publ. Eur. Soc. Clin. Microbiol. Infect. Dis. 2018, 24, 175-179. [CrossRef]

22. Jayol, A.; Nordmann, P.; Brink, A.; Villegas, M.-V.; Dubois, V.; Poirel, L. High-Level Resistance to Colistin Mediated by Various Mutations in the crrB Gene among Carbapenemase-Producing Klebsiella pneumoniae. Antimicrob. Agents Chemother. 2017, 61. [CrossRef] [PubMed]

23. Donà, V.; Bernasconi, O.J.; Kasraian, S.; Tinguely, R.; Endimiani, A. A SYBR ${ }^{\circledR}$ Green-based real-time PCR method for improved detection of mcr-1-mediated colistin resistance in human stool samples. J. Glob. Antimicrob. Resist. 2017, 9, 57-60. [CrossRef] [PubMed]

24. Osei Sekyere, J. Mcr colistin resistance gene: A systematic review of current diagnostics and detection methods. Microbiologyopen 2019, 8, e00682. [CrossRef] [PubMed]

25. Stefaniuk, E.M.; Tyski, S. Colistin Resistance in Enterobacterales Strains-A Current View. Polish J. Microbiol. 2019, 68, 417-427. [CrossRef]

26. Osei Sekyere, J.; Govinden, U.; Bester, L.A.; Essack, S.Y. Colistin and tigecycline resistance in carbapenemase-producing Gramnegative bacteria: Emerging resistance mechanisms and detection methods. J. Appl. Microbiol. 2016, 121, 601-617. [CrossRef] [PubMed]

27. Chew, K.L.; La, M.-V.; Lin, R.T.P.; Teo, J.W.P. Colistin and Polymyxin B Susceptibility Testing for Carbapenem-Resistant and mcr-Positive Enterobacteriaceae: Comparison of Sensititre, MicroScan, Vitek 2, and Etest with Broth Microdilution. J. Clin. Microbiol. 2017, 55, 2609-2616. [CrossRef] [PubMed]

28. Flores-Treviño, S.; Garza-González, E.; Mendoza-Olazarán, S.; Morfín-Otero, R.; Camacho-Ortiz, A.; Rodríguez-Noriega, E.; Martínez-Meléndez, A.; Bocanegra-Ibarias, P. Screening of biomarkers of drug resistance or virulence in ESCAPE pathogens by MALDI-TOF mass spectrometry. Sci. Rep. 2019, 9, 18945. [CrossRef]

29. Charretier, Y.; Schrenzel, J. Mass spectrometry methods for predicting antibiotic resistance. Proteomics. Clin. Appl. 2016, 10, 964-981. [CrossRef]

30. Calderaro, A.; Buttrini, M.; Piergianni, M.; Montecchini, S.; Martinelli, M.; Covan, S.; Piccolo, G.; Medici, M.C.; Arcangeletti, C.; Chezzi, C.; et al. Evaluation of a modified meropenem hydrolysis assay on a large cohort of KPC and VIM carbapenemaseproducing Enterobacteriaceae. PLoS ONE 2017, 1-12. [CrossRef] [PubMed] 
31. Furniss, R.C.D.; Dortet, L.; Bolland, W.; Drews, O.; Sparbier, K.; Bonnin, R.A.; Filloux, A.; Kostrzewa, M.; Mavridou, D.A.I.; Larrouy-Maumus, G. Detection of Colistin Resistance in Escherichia coli by Use of the MALDI Biotyper Sirius Mass Spectrometry System. J. Clin. Microbiol. 2019, 57. [CrossRef] [PubMed]

32. Dortet, L.; Potron, A.; Bonnin, R.A.; Plesiat, P.; Naas, T.; Filloux, A.; Larrouy-Maumus, G. Rapid detection of colistin resistance in Acinetobacter baumannii using MALDI-TOF-based lipidomics on intact bacteria. Sci. Rep. 2018, 8, 16910. [CrossRef] [PubMed]

33. Liao, W.; Lin, J.; Jia, H.; Zhou, C.; Zhang, Y.; Lin, Y.; Ye, J.; Cao, J.; Zhou, T. Resistance and Heteroresistance to Colistin in Escherichia coli Isolates from Wenzhou, China. Infect. Drug Resist. 2020, 13, 3551-3561. [CrossRef] [PubMed]

34. Croxatto, A.; Prod'hom, G.; Greub, G. Applications of MALDI-TOF mass spectrometry in clinical diagnostic microbiology. FEMS Microbiol. Rev. 2012, 36, 380-407. [CrossRef]

35. Calderaro, A.; Buttrini, M.; Martinelli, M.; Farina, B.; Moro, T.; Montecchini, S.; Arcangeletti, M.C.; Chezzi, C.; De Conto, F. Rapid classification of clostridioides difficile strains using maldi-tof ms peak-based assay in comparison with pcr-ribotyping. Microorganisms 2021, 9, 661. [CrossRef]

36. Calderaro, A.; Motta, F.; Montecchini, S.; Gorrini, C.; Piccolo, G.; Piergianni, M.; Buttrini, M.; Medici, M.C.; Arcangeletti, M.C.; Chezzi, C.; et al. Identification of Dermatophyte Species after implementation of the in-house MALDI-TOF MS database. Int. J. Mol. Sci. 2014, 15, 16012-16024. [CrossRef] [PubMed]

37. Calderaro, A.; Gorrini, C.; Piccolo, G.; Montecchini, S.; Buttrini, M.; Rossi, S.; Piergianni, M.; Arcangeletti, M.C.; De Conto, F.; Chezzi, C.; et al. Identification of Borrelia species after creation of an in-house MALDI-TOF MS database. PLoS ONE 2014, 9. [CrossRef] [PubMed] 